\title{
Surgical Management of Unroofed Coronary Sinus Syndrome: A 20-Year-Single-Center Experience In 159 Patients
}

\author{
Hao Shi ${ }^{1}$, Jun Yan ${ }^{1}$, Qiang Wang ${ }^{1}$, Zhongdong Hua ${ }^{1}$, Shoujun Li $^{1}$, and Jing Zhang ${ }^{2}$ \\ ${ }^{1}$ Chinese Academy of Medical Sciences \& Peking Union Medical College Fuwai Hospital \\ ${ }^{2}$ Chinese Academy of Medical Sciences and Peking Union Medical College Fuwai Hospital
}

April 28, 2020

\begin{abstract}
Background. Unroofed coronary sinus syndrome (UCSS) is rare and often associated with Left superior vena cava (LSVC). We report our experience in 159 patients with UCSS during a 20-year period in terms of clinical features, diagnosis, associated anomalies, surgical procedures and late outcomes. Methods. Between May 1998 and May 2019, 159 patients with UCSS were treated surgically and followed up. UCSS was confirmed by preoperative echocardiography or CT scan in 97 patients and by the surgeons during surgery in 62 . LSVC directly drained into the left atrium (LA) was found in 100 cases. In these patients, 8 cases of LSVC were ligated, 59 cases were reconstructed the intracardiac tunnel to drain LSVC to right atrium (RA), and the extracardiac procedure was constructed to lead the LSVC draining to RA in 2. The associated cardiac lesions were corrected concomitantly. Results There were 5 hospital deaths. We followed up 143 early survivors, and there was no death. Except for one case of avulsion of the patch in which LSVC was drained by internal tunnel, there were no serious complications in other followup patients. Conclusion. UCSS is often misdiagnosed in the preoperative evaluation of congenital heart diseases. Preoperative transthoracic echocardiography (TTE) is still the most important method in the diagnosis of UCSS. When associated with LSVC, UCSS should be considered as a possible additional finding. We performed different surgical approaches to deal with the different types of UCSS with LSVC with a good result.
\end{abstract}

\section{Surgical Management of Unroofed Coronary Sinus Syndrome: A 20-Year-Single-Center Expe- rience In 159 Patients}

Running head Unroofed Coronary Sinus Syndrome

Hao Shi, Jun Yan, Qiang Wang, Zhongdong Hua, Shoujun Li, Jing Zhang

Department of Pediatric Cardiac Surgery, National Center for Cardiovascular Disease and Fuwai Hospital, Chinese Academy of Medical Sciences, Peking Union Medical College, 167 Beilishi Road, Xicheng, Beijing 100037, People's Republic of China

Corresponding author: Jing Zhang. E-mail:zhangjingfw@163.com.

\section{【Abstract 】}

Background . Unroofed coronary sinus syndrome (UCSS) is rare and often associated with Left superior vena cava (LSVC). We report our experience in 159 patients with UCSS during a 20-year period in terms of clinical features, diagnosis, associated anomalies, surgical procedures and late outcomes.

Methods. Between May 1998 and May 2019, 159 patients with UCSS were treated surgically and followed up. UCSS was confirmed by preoperative echocardiography or CT scan in 97 patients and by the surgeons during surgery in 62 . LSVC directly drained into the left atrium (LA) was found in 100 cases. In these patients, 8 cases of LSVC were ligated, 59 cases were reconstructed the intracardiac tunnel to drain LSVC 
to right atrium (RA), and the extracardiac procedure was constructed to lead the LSVC draining to RA in 2. The associated cardiac lesions were corrected concomitantly.

Results There were 5 hospital deaths. We followed up 143 early survivors, and there was no death. Except for one case of avulsion of the patch in which LSVC was drained by internal tunnel, there were no serious complications in other follow-up patients.

Conclusion. UCSS is often misdiagnosed in the preoperative evaluation of congenital heart diseases. Preoperative transthoracic echocardiography (TTE) is still the most important method in the diagnosis of UCSS. When associated with LSVC, UCSS should be considered as a possible additional finding. We performed different surgical approaches to deal with the different types of UCSS with LSVC with a good result.

【Key words \Unroofed coronary sinus; Cardiac surgical procedures; Left superior vena cava; Echocardiography; Congenital heart disease

\section{Introduction}

UCSS is a group of rare, complex cardiac malformations caused by the absence of left atrial venous plica during the development of the embryo, that is, the partial or complete defect of coronary sinus (CS) septum, which lead to direct communication between CS and the LA. UCSS is often associated with LSVC (80\% to $90 \%$ ), whose overall incidence is about $0.3 \%$ to $0.5 \%$, and incidence in congenital heart diseases is about $3 \%$ to $10 \%[1]$. Preoperative diagnosis used to be difficult because of the lack of specific clinical manifestations and examination methods of UCSS. From May 1998 to May 2019, we treated 68740 cases of congenital heart diseases, including 159 cases of UCSS (0.23\%), which treated by different methods of operation and achieved good effects. This paper is to summarize the clinical characteristics and surgical treatment experience, and to provide reference for the preoperative diagnosis and treatment of such diseases in the operation, in order to improve the curative effect

\section{Methods \\ Patient Profiles}

There were 67 males and 92 females. Their mean age was $15.7 \pm 17.2$ (range 0.3-59) years and median body weight was 31.5 \pm 25.1 (range 3.4-85) kg. According to the morphologic type of UCSS classified as Kirklin and Barratt-Boyes reported[2]: type I (completely unroofed with LSVC, Fig 1A ) 76 cases; type II (completely unroofed without LSVC) 24 cases; type III (partially unroofed midportion) 34 cases; type IV (partially unroofed terminal portion) 25 cases. Preoperative transthoracic echocardiography (TTE) were performed in all cases, and 91 cases of all patients were confirmed by TTE preoperatively, while the remaining 6 patients diagnosed before operation were found by CT scan. Among the 97 cases, 46 cases were type I, 16 were type II, 21 were type III, 14 were type IV. The remaining 62 cases were diagnosed by intraoperative exploration. Preoperative TTE confirmed 71 cases of LSVC (53 cases of type I, 14 cases of type III, 4 cases of type IV), and 29 cases of LSVC were found by intraoperative exploration. The clinical classification and associated cardiac malformations in 159 patients with UCSS are shown in Table 1.

\section{Operative Technique}

The operations were performed under general anesthesia and hypothermic cardiopulmonary bypass. 145 cases underwent thoracotomy through the median incision of the chest, 10 cases were admitted to the chest through the right incisions, and 4 cases were admitted into the chest through infrasternal small incisions. If LSVC exists, we would compare the diameter of bilateral superior vena cava and block LSVC. If the LSVC pressure is greater than $16 \mathrm{mmHg}$ or the left side of the neck appears cyanosis or venous engorgement, it is positive result showing that there is no communication between LSVC and right superior vena cava (RSVC), and LSVC can't be ligated. LSVC drainage is necessary for surgeons to establish extracorporeal circulation. If the block test result is negative, the LSVC can be ligated. UCSS and LSVC and other associated intracardiac malformations will be treated simultaneously during the surgery. 
Type I 76 cases: 58 cases constructed the inner tunnel(Fig 1B、C) along the posterior wall of the LA, and LSVC was drained to the CS, and the atrial septum was closed with patch around the new ostium of the CS. Four cases had no significant pressure increase of central veins or edema of head and face after obstructing the LSVC, so their LSVCs were directly ligated, and the CS was separated into the LA by the atrial septal patch. Nine cases underwent atrial septal resection, and then the large autologous pericardium (or polyester patch) were slantwise arranged in the opened left and right atrium to create a baffle, separating the LSVC to the right atrial side and the left atrial appendage, mitral valve and all pulmonary veins to the left atrial side. Three cases of direct CS reroof by pericardium. One case used bovine pericardium to make extracardiac conduit and connected LSVC and right atrial appendage and another extracardiac case underwent Glenn procedure.

Type II 24 cases: 5 cases of atrial septal patch separated the CS into the LA and 13 cases into RA. Six cases of direct CS reroof by pericardium.

Type III 34 cases: 16 cases were combined with LSVC, 1 of which was directly ligated. One case took extracardiac method to connect LSVC to the right atrium. Three cases of LSVC were treated by intracardiac tunnel or baffle and the rest were treated under the reroof procedures.

Type IV 25 cases: 8 cases were combined with LSVC ( 6 cases were drained to the CS, 2 cases to the LA), among which 3 cases were directly ligated, 2 cases were reroofed the CS, and 3 separated the CS opening to the right atrium by the atrial septal patch.

In 159 cases associated with other intracardiac malformations, 2 cases underwent palliative surgery due to complex malformations, 157 cases underwent simultaneous correction of associated malformations. All 159 patients with LSVC and intraoperative treatment are shown in Table 2.

\section{Results}

The mean cardiopulmonary bypass time was $128 \pm 73.9 \min \left(34^{\sim} 563 \mathrm{~min}\right)$. The mean aortic occlusion time was $86.5 \pm 46.3 \mathrm{~min}\left(18^{\sim} 255 \mathrm{~min}\right)$. There were 5 cases of operative death, 4 cases of type I, 1 cases of type IV, all of which were combined with LSVC.

Severe postoperative complications mainly included severe lung infection, perfusion lung, diaphragmatic paralysis and anuria in 4 cases. In addition, 1 case of type I UCSS with RSVC deficiency and transitional endocardial cushion defect was treated with pericardial piece to establish an internal tunnel to drain LSVC. The avulsion of pericardial piece and narrow opening of internal tunnel were found by ultrasound after operation, and the opening of RSVC was found by ultrasound 3 months after operation.

The other 149 cases recovered smoothly in early postoperative period, with a mean mechanical ventilation time for $13.8 \pm 11.7 \mathrm{~h}\left(2^{\sim} 63 \mathrm{~h}\right)$. Their mean postoperative hospital stay was $9.3 \pm 5.4$ days $\left(5^{\sim} 36\right.$ days $)$. There was no arrhythmia after operations, and TTE examination before discharge showed no tunnel stenosis, marginal residual shunt and pulmonary venous obstruction except the case above.

Among the survivors, 143 cases were followed up for 2 months to 18 years. One case had postoperative residual ASD shunt, and 2 cases were newly diagnosed with discrete subaortic membranes 3 years after the operation, 1 of which underwent surgical treatment. All cases received intracardiac treatment with LSVC occurred no death and complications after operations.

\section{Discuss}

UCSS is rare and often associated with LSVC. There are 62.9\% (100/159) patients with LSVC in our series. The hemodynamic changes of UCSS depend on: (1) the presence of LSVC; (2) whether the CS opening is expanded, constricted or occluded; (3) whether there is a communication between the CS and the LA; (4) whether there are associated malformations such as the blood flow obstruction in the left or right atrioventricular system[3]. In our series, only 18 cases were sole UCSS, the remaining 141 cases were associated with other intracardiac malformations, the most common ones were associated ECD (40.9\%, 
65/159). Most clinical manifestations of UCSS should be analyzed comprehensively in combination with other associated cardiac malformations.

\section{Preoperative diagnosis}

The clinical manifestation of UCSS is lack of specificity, which makes it difficult to diagnose before and during the operation. In our series, 91 patients $(57.2 \%)$ were diagnosed by TTE before operation, and 6 patients $(3.77 \%)$ were diagnosed by CT. Before 2008, only 14 of the 46 cases were preoperative diagnosed as UCSS (30.4\%), and after 2008,81 of $113(71.7 \%)$ cases were diagnosed before operation. It was precisely because of the increased understanding of the pathological anatomy and pathophysiological features of UCSS, the rate of preoperative diagnosis of UCSS in our hospital has improved significantly in recent years. It was reported that the accuracy of UCSS diagnosis by preoperative TTE and acoustic angiography was $65 \%$ [4], and the diagnosis rate of this series was quite similar after 2008.

TTE examination was easy to detect CS expansion, and suprasternal fossae scanning was easy to make it clear the presence of LSVC and the communication via LIV and RSVC, while $80 \%$ 90\% LSVC patients without communications [5]. If the ultrasound didn't detect the CS, it suggested completely unroofed type I or type II. Therefore, careful attention should be paid to TTE or intraoperative exploration to avoid missed diagnosis. Cardiac catheterization examination via the left upper limb venous catheterization was easy to find LSVC; if catheterization via the right upper limb vein and catheter tip through the right atrium and then appeared in the left superior mediastinum, it also suggested the presence of LSVC. It was reported that it was more likely to diagnose UCSS using transesophageal echocardiography (TEE), real-time threedimensional echocardiography, or magnetic resonance imaging (MRI)[6-8]. In recent years, several cases of TTE diagnosis errors have been reported, which have been diagnosed as UCSS by TEE, MDCT or MRI [9-11].

Although preoperative diagnostic rates have improved markedly in recent years, careful intraoperative intracardiac and extracardiac inspection remains critical. In our series, 62 cases (37.1\%) were diagnosed by intraoperative inspection. If it is found in intraoperative extracardiac inspection that LSVC does not converge to the CS but directly into the LA, the malformation should be considered. If the expansion of CS is detected in the right atrium, or red blood flows out of it, or the CS opening can't be found, we should think more of the presence of this malformation. When necessary, open the atrial septum and inspect the left and right atrium together to diagnose [12].

\section{Operative treatment of LSVC}

The surgical treatment of UCSS depend on the type of the CS septal defect, but depend more on the presence of LSVC and the communication between RSVC and LSVC, that is, more likely based on whether LSVC can be ligated. The ligation criteria in this series of patients was that when obstructing the LSVC, the clamped pressure was not higher than $16 \mathrm{mmHg}$, and no cyanosis and venous engorgement occurred in the left side of the neck, then LSVC can be ligated. There were reports of the ligation when central venous pressure was less than $30 \mathrm{mmHg}$, although right upper limbs and fontanelle edema occurred in early postoperative period. The patients recovered in a few weeks, and had no neurological complications [13].For those who have UCSS without LSVC or with LSVC that can be ligated, it only need to repair ASD and close the CS opening, or separate the CS opening to the LA when repairing the ASD by the patch, which resulted in a small amount of right-to-left shunt that had no conspicuous physiological effects. The right-to-left shunt caused by pure UCSS only accounted for $4 \%$ to $5 \%$ of the total blood flow. The impact was very small, and no symptoms or cyanosis occurred. In our series, 8 cases (type I 4 cases, type III 1 case, type IV 3 cases) underwent direct ligation of LSVC, the method is simple and effective, and no complications occurred after operation.

When associated with LSVC that can't be ligated, the LSVC is rerouted to the right atrium, and different types of CS septal defect require should be treated differently: (1) Intracardiac treatment: baffling or tunneling in the LA to drain the LSVC to the right atrium, and repair the septal defect [Fig 2]; (2) Extracardiac treatment: there are several ways: LSVC-right atrium connection, LSVC-RSVC connection, LSVC-left pulmonary artery connection[14-16]. The intracardiac treatment is the most classic method. Of the series 59 
cases with UCSS underwent the left atrial tunnel, 22 intracardiac baffle repair, 4 of which died after the operations, all associated with complex cardiac abnormalities and severe pulmonary hypertension and died of postoperative pulmonary infection. Sixty-five cases were followed up after the operation, no death and complications occurred. In the case of LSVC returning directly to the LA, LSVC often joins the LA anterior to the left atrial appendage and the left pulmonary vein. The left pulmonary vein opening should be noted during the operation so as to avoid obstruction of the left pulmonary vein. Intracardiac treatment required a long operation time, with long duration of myocardial ischemia and postoperative complications such as baffle residual shunt, patch damage, LSVC reflux obstruction, pulmonary venous obstruction, left ventricular inflow tract obstruction and so on. In addition, the disadvantages of intracardiac approach are more obvious for complex congenital heart disease or younger children with small LA. At this point, some authors believe that an extracardiac approach might be better for correcting LSVC ectopic drainage [14]. When LSVC is fully dissociated, connect it to the right atrium or RSVC through the anterior or posterior aorta. Gore -Tex artificial blood vessels have also been reported to successfully connect the LSVC to the right atrium [15]. However, this method is not commonly used, because it may cause obstruction of the superior vena cava or obstruction of the artificial blood vessel after operation which result from the compression of the large vessels or the sternum.

The surgical indication of LSVC connection with the left pulmonary artery is the same as bidirectional Glenn shunt, and for cases associated with pulmonary hypertension, this procedure is not appropriate. Therefore, to deal with cases of LSVC directly drained to the LA, we would highly recommend the choice of intracardiac method of rerouting LSVC, if the surgery is found difficult or ineffective, then the extracardiac treatment would be an alternative method.

The operative effect of intracardiac tunnel in our series is good. The key lies in careful design and operative techniques, and it is still the mainstream treatment, while the external cardiac method is suitable for few cases, so we should choose carefully.

\section{Conclusion}

Congenital heart disease with LSVC should be aware of the presence of UCSS. Preoperative TTE is still the main method to diagnose UCSS, and it can improve the diagnostic rate of TTE to strengthen the understanding of the pathologic anatomy and pathophysiological characteristics of this cardiac malformations. According to the location of LSVC into the LA, different surgical methods were selected, and satisfactory results were achieved. The mortality rate of this series of patients was $3.1 \%(5 / 159)$, the cause of death mainly depends on the complexity and severity of the associated cardiac malformations.

\section{Limitations}

There were several limitations to this study. Firstly, because all the death occurred in associated complex anomalies, and the short-term and long-term results of the surviving patients were good, no statistical correlation study was conducted in this study. Secondly, all follow-up medical examinations were mainly echocardiography. We did not carry out CT or MRI examination for patients with intracardiac tunnel or extracardiac method, which can show the operation effects more clearly.

\section{Acknowledgements}

We acknowledge the roles of our colleagues, perfusionists, nurses, and others involved in the care of the study patients.

\section{Funding}

This study was supported by the National Key R\&D Program of China [2017YFC1308100].

\section{Compliance with Ethical Standards}

Conflict of interest The authors declare that they have no conflicts of interest. 
Ethical Approval All procedures performed in studies involving human participants were in accordance with the ethical standards of the institutional and national research committee and with the 1964 Helsinki Declaration and its later amendments or comparable ethical standards.

Informed Consent Informed consent was obtained from all patients, parents, or guardians included in the study.

\section{Reference}

1. Cristina Giuliani-Poncini, Marie-Helene Perez, Jacques Cotting, et al. Persistent Left Superior Vena Cava in Cardiac Congenital Surgery[J]. Pediatric Cardiology, 2014, 35(1):71-76. DOI:10.1007/s00246013-0743-z.

2. Kirklin JW, Barratt-Boyes BG.Unroofed coronary sinus syndrome//Kirklin JW, Barratt-Boyes BG.Cardiac surgery[M].2nd ed.New York: Churchill-Livingstone, 1993: 683-692.

3. Zhang J, Sun HS, Luo XJ, et al.Surgical treatment of unroofed coronary sinus syndrome[J].Chin J Clin Thorac Cardiovasc Surg,2008,15(6):402405. DOI: CNKI:SUN:ZXYX.0.2008-06-004

4. Xie MX, Yang YL, Cheng TO, et al.Coronary sinus septal defect (unroofed coronary sinus): Echocardiographic Diagnosis and Surgical Treatment[J].International Journal of Cardiology, 2013,168(2):12581263. DOI: $10.1016 /$ j.ijcard.2012.11.113.

5. Shingo Chihara, Hiroshi Yasunaga, Kageshige Todo. Anastomosis of left to right superior vena cava for repair of Unroofed Coronary Sinus[J]. General Thoracic and Cardiovascular Surgery, 2012, 60(4):p.244246. DOI: $10.1007 / \mathrm{s} 11748-011-0815-\mathrm{z}$.

6. Guo R, Sun JP, Lee AP, et al.A rare case with unroofed coronary sinus defect and aneurysmal midcardiac vein[J].Int J Cardiol.2014,177(3): e158-e160. DOI:10.1016/j.ijcard.2014.08.149.

7. H Kim, Y H Choe, S W Park, et al. Partially Unroofed Coronary Sinus: MDCT and MRI Findings[J]. AJR American Journal of Roentgenology, 2010, 195(5). W331-W336. DOI:10.2214/AJR.09.3689.

8. Disha B , Prakashini K, Shetty R K . Persistent left superior vena cava in association with sinus venosus defect type of atrial septal defect and partial pulmonary venous return on 64-MDCT[J]. BMJ Case Reports, 2014, 2014(May21 2). DOI: 10.1136/bcr-2013-202999.

9. Snijder R J R, Schaap J, Verzijlbergen J F, et al. Imaging of an unusual case of a completely unroofed coronary sinus without persistent left superior vena cava $[\mathrm{J}]$. European Heart Journal Cardiovascular Imaging, 14(3):297-297. DOI: 10.1093/ehjci/jes190.

10. Yoshinori Miyahara, Koichi Kataoka, Masaaki Kawada. Isolated Unroofed Coronary Sinus on ThreeDimensional Computed Tomographic Imaging[J]. Annals of Thoracic Surgery, 2012, 93(6): 2072. DOI: 10.1016/j.athoracsur.2011.11.015.

11. Abhishek Chaturvedi, Theodore J. Dubinsky, Jeffrey H. Maki. MR findings of a rare defect, coronary sinus ASD [J]. International Journal of Cardiovascular Imaging, 2012, 28(2):429-430. DOI: 10.1007/s10554010-9670-8.

12. Jing Zhang, Zhi-Xiong Huang, Han-Song Sun, et al. Clinical analysis of unroofed coronary sinus syndrome with atrioventricular canal defect[J]. Chin J Surg, 2009, 47(11): 845-848.

13. Zimand S, Benjamin P, FrandM, et al.Left superior vena cava to the left atrium: Do we have to change the traditional approach? [J].Ann Thorac Surg,1999,68(5):1869-1872. DOI: 10.1016/S00034975(99)01037-1.

14. Muthialu Nagarajan, Fajardo Diana, Sullivan Ian D, et al. Repair of Persistent Left Superior Vena Cava to Unroofed Coronary Sinus Defect by Retro-Aortic Implantation (Modified Warden Type Procedure) [J]. Journal of Cardiac Surgery, 2016, 31(2):103-105. DOI: 10.1111/jocs.12684.

15. Gontigo B, Fantini EA, de Paula e Lilva, et al. The use of PTFE graft to correct anomalous drainage of persistent left superior vena cava. J Cardiovasc Surg(Torino), 1990, 31: 815-817. DOI: 10.1097/00019501-199011000-00014.

16. Andrea Quarti,Marco Di Eusanio, et al. Left Superior Vena Cava Draining into the Left Atrium, Associated with Partial Anomalous Pulmonary Venous Connection: Surgical Correction. Journal of Cardiac Surgery,2005,20(4):353-355. DOI; 10.1111/j.1540-8191.2005.200471.x. 


\section{Figure legends}

Fig 1 Treatment of type I UCSS by constructing internal tunnel: A. Preoperative TTE showed that LSVC drained into LA and CS was totally unroofed, associated with single atrium. B. Postoperative TEE showed drainage of LSVC to CS by establishing intracardiac tunnel. C. During the operation, a straight drainage tube was inserted into LSVC directly and the internal tunnel(arrow) was anastomosed along the tube.

Fig 2 Intracardiac treatment of LSVC drained into LA. A. Intracardiac baffle. B. Intracardiac tunnel.

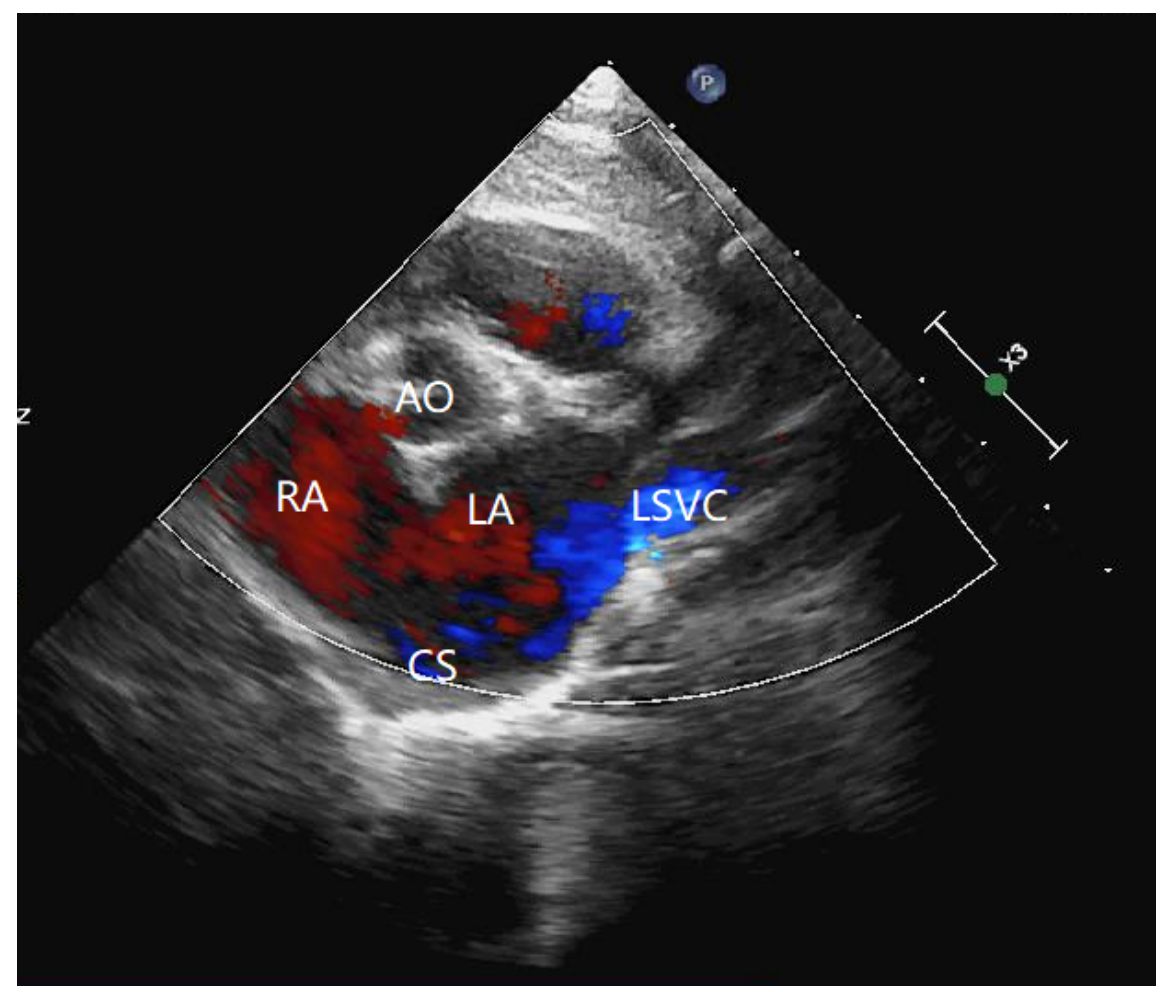



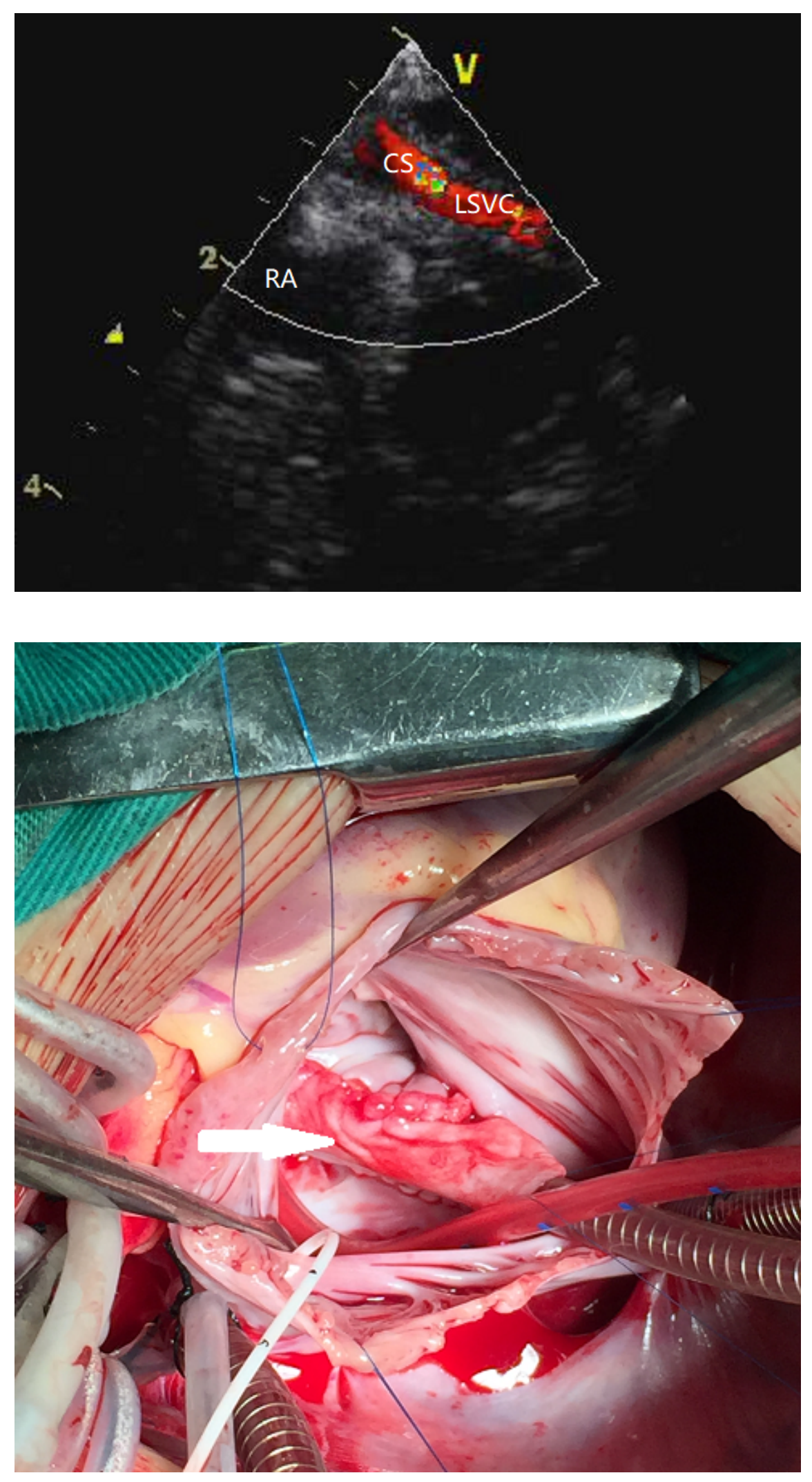

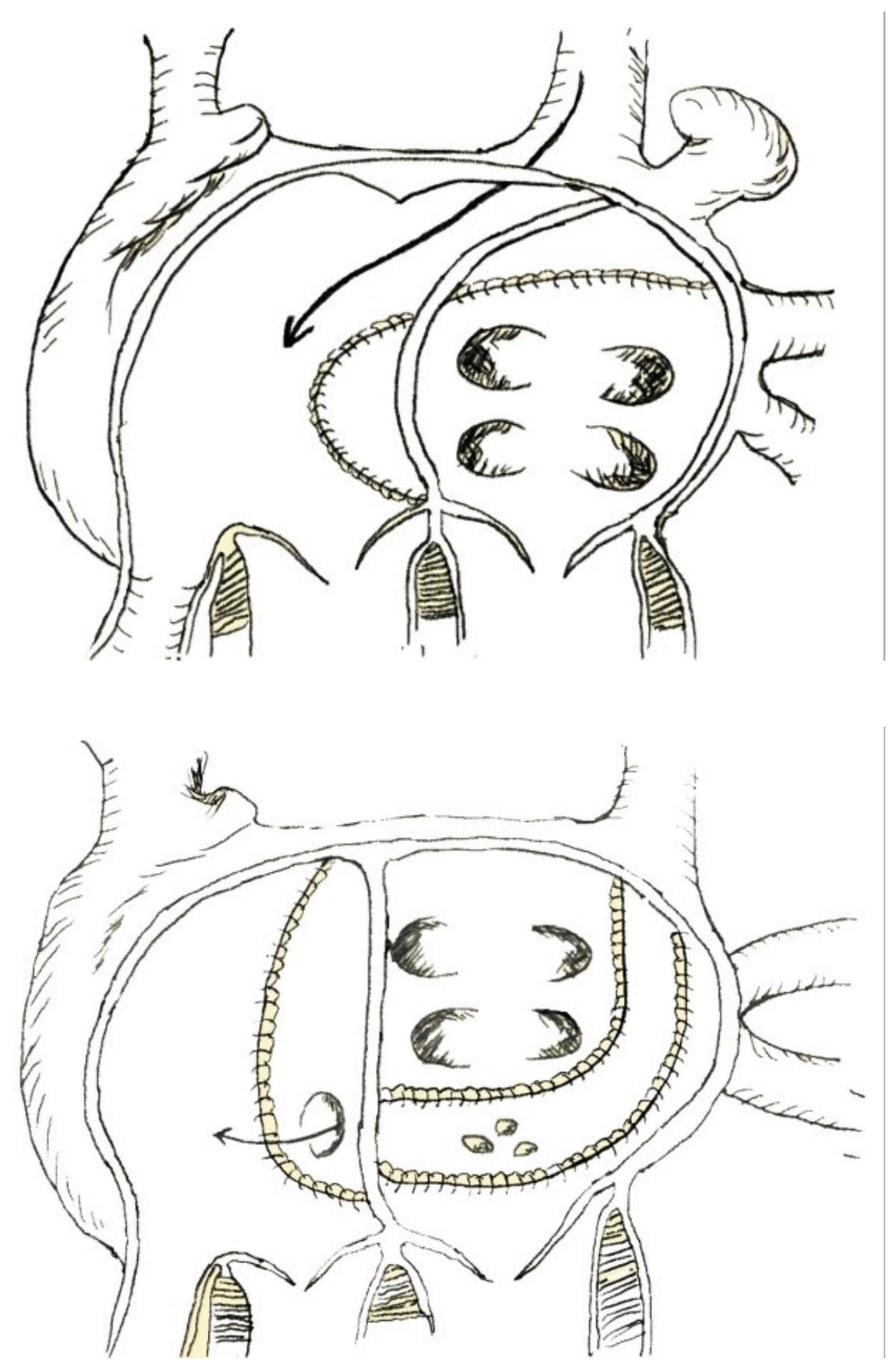

\section{Hosted file}

Table 1. docx available at https://authorea.com/users/313725/articles/444165-surgical-managementof-unroofed-coronary-sinus-syndrome-a-20-year-single-center-experience-in-159-patients

\section{Hosted file}

Table 2.docx available at https://authorea.com/users/313725/articles/444165-surgical-managementof-unroofed-coronary-sinus-syndrome-a-20-year-single-center-experience-in-159-patients 